\title{
Efectos de la expresión anormal de miR-16 y miR-1296 en la supervivencia global de pacientes adultos colombianos con leucemia linfoblástica aguda tratados con el esquema Hyper CVAD
}

- Leonardo Enciso, Ismael Samudio, Antonio Huertas, Martha Lucía Díaz, Martha Suárez, Natalia Olaya, Sebastián Alcalá

Programa de Investigación en Leucemia Aguda y Crónica (Pilac), Instituto Nacional de Cancerología, Bogotá, D.C. Contacto: ljeo2812@hotmail.com

\begin{abstract}
Introducción y objetivos. La expresión de microRNAs y sus efectos biológicos han sido identificados como variables pronósticas en pacientes con diferentes tipos de tumor, pero sus efectos en los pacientes adultos con LLA no han sido bien caracterizados. El objetivo de este estudio fue describir el perfil de expresión de miRNAs en pacientes adultos con LLA atendidos en nuestro centro y determinar su efecto en la supervivencia global.

Materiales y métodos. Estudio de cohorte prospectivo que incluye pacientes adultos con diagnóstico nuevo de LLA. El análisis de la expresión de miRNAs se realizó mediante PCR cuantitativa en tiempo real. Se efectuó un agrupamiento supervisado y se definieron los miRNAS diferencialmente expresados ajustando los valores de $p$ para pruebas multivariadas. La OS y PFS fueron estimadas por el método de Kaplan-Meir.
\end{abstract}

Resultados. Se incluyeron 30 pacientes y tres controles. Tanto el hsa-miR-1296 como el hsa-miR-16 fueron encontrados expresados de manera diferencial entre grupos. Basados en las interacciones miRNA-mRNA predichas y sus efectos biológicos conocidos, se determinó el efecto en la OS de la expresión de estos dos miRNAs, haIlando que el grupo con expresión de hsa-miR-1296 y hsa-miR-16 por arriba del punto de corte tuvo una supervivencia global inferior al grupo con valores de expresión inferiores, y estas diferencias fueron estadísticamente significativas $-p=0,047$ y $p=0,04$ (log-rank)-. De los 12 pacientes que murieron durante el período de seguimiento, 10 (83,3\%) pertenecían al grupo miR-1296 alto.

Conclusiones. Los pacientes con una expresión de miR-1296 y miR-16 mayor al punto de corte tienen una menor probabilidad de supervivencia.

\section{IL-8 en el microambiente tumoral de leucemia linfoide aguda B en médula ósea y sangre periférica se asocia con recuentos elevados de células T reguladoras, con el grado de infiltración}

Sandra Quijano Gómez, Diana Carolina Anaya Revolledo, Sandra Paola Santander González, lliana de los Reyes Valencia,

María Victoria Herrera Jauregui, Julio Solano Vega, Susana Fiorentino Gómez

Grupo de Inmunobiología y Biología Celular, Departamento de Microbiología, Pontificia Universidad Javeriana,

Centro Javeriano de Oncología, Hospital Universitario San Ignacio, Bogotá, D.C.

Contacto: squijano@javeriana.edu.co

Introducción y objetivos. La presencia de linfocitos $T$ reguladores (Tregs) en el microambiente tumoral y la expresión del factor de transcripción FOXP3 se asocian con mal pronóstico. Existen pocos estudios que evalúen la presencia de Tregs y de FOXP3 en leucemia linfoide aguda B (LLA-B). Objetivo: describir la frecuencia de Tregs en médula ósea (MO) y sangre periférica (SP), su asociación con el perfil de citoquinas solubles en plasma y con la expresión de CXCR1 (receptor de IL-8), y analizar la expresión de FOXP3 en los blastos de pacientes con LLA-B respecto a controles sanos.

Materiales y métodos. En total se evaluaron $20 \mathrm{MO}$ y SP de pacientes con LLA-B y $10 \mathrm{MO}$ y SP de controles. El análisis de Tregs, citoquinas y FOXP3 se realizó por citometría de flujo.
Resultados. En las LLA-B se observó un incremento significativo en las frecuencias de Tregs en MO y SP, siendo superiores en SP en comparación con la MO. Los recuentos absolutos de Tregs se asociaron con los recuentos absolutos de blastos en $\mathrm{MO}$ y SP, y con la concentración de IL-8 soluble. Las concentraciones de IL-8 e IL-6 se relacionaron con la expresión de CXCR1 en los blastos. Además, en los blastos de LLA-B se observa mayor expresión de FOXP3 en comparación con los precursores linfoides normales.

Conclusiones. Estos resultados sugieren que en las LLA-B las Tregs y el microambiente de citoquinas pueden representar mecanismos de evasión de la respuesta inmune, por lo que es importante hacer seguimiento de los pacientes para ver su impacto en el pronóstico. 